\title{
A theoretical analysis of time-dependent fragment momenta in indirect photofragmentation
}

\section{Henriksen, Niels Engholm}

Published in:

Journal of Chemical Physics

Link to article, DOI:

$10.1063 / 1.3455997$

Publication date:

2010

Document Version

Publisher's PDF, also known as Version of record

Link back to DTU Orbit

Citation (APA):

Henriksen, N. E. (2010). A theoretical analysis of time-dependent fragment momenta in indirect

photofragmentation. Journal of Chemical Physics, 132(23), 234311. https://doi.org/10.1063/1.3455997

\section{General rights}

Copyright and moral rights for the publications made accessible in the public portal are retained by the authors and/or other copyright owners and it is a condition of accessing publications that users recognise and abide by the legal requirements associated with these rights.

- Users may download and print one copy of any publication from the public portal for the purpose of private study or research.

- You may not further distribute the material or use it for any profit-making activity or commercial gain

- You may freely distribute the URL identifying the publication in the public portal

If you believe that this document breaches copyright please contact us providing details, and we will remove access to the work immediately and investigate your claim 


\title{
A theoretical analysis of time-dependent fragment momenta in indirect photofragmentation
}

\author{
Niels E. Henriksen ${ }^{\text {a) }}$ \\ Department of Chemistry, CMM, Technical University of Denmark, Building 207, \\ DK-2800 Kgs. Lyngby, Denmark
}

(Received 22 March 2010; accepted 2 June 2010; published online 18 June 2010)

\begin{abstract}
We study theoretically diatomic molecules which are prepared in a superposition of quasibound resonance states by a femtosecond laser pulse. An analytical (Landau-Zener-like) result is derived for the momentum distribution of the atomic fragments in the asymptotic force-free region after a single passage of a curve crossing. Furthermore, at later times, simple analytical expressions show how the emerging structures in the momentum distribution are related to the energies of the resonance states. (0) 2010 American Institute of Physics. [doi:10.1063/1.3455997]
\end{abstract}

\section{INTRODUCTION}

In unimolecular fragmentation reactions activated by a femtosecond laser pulse, the temporal evolution of the process where the fragments fly apart can basically take two different forms: either the fragmentation is essentially instantaneous, within a typical vibrational period following the activation, or products may be formed over an extended period of time of many vibrational periods. Thus, the fragmentation can be direct and as soon as the final products are formed, i.e., the fragments have entered the force-free region, the distribution over chemical channels as well as the population of the associated quantum states is fixed. In indirect fragmentation, an intermediate energized complex is formed. In principle, all products can then be formed more or less instantaneously after a delay, or products may continuously "leak out" of the complex over an extended period of time of many vibrational periods. In this paper, we consider the latter situation.

The experimental and theoretical work on the femtosecond dynamics of $\mathrm{NaI}$ is a benchmark illustration of indirect unimolecular dynamics, ${ }^{1-4}$

$$
\stackrel{\text { pump }}{\mathrm{NaI} \rightarrow(\mathrm{NaI})^{*} \rightarrow \mathrm{Na}+\mathrm{I} .}
$$

The vibrational period of the quasibound state of this molecule, (NaI)* see Fig. 1, is around 1 ps when the molecule is excited at $328 \mathrm{~nm}$. The buildup of the total population of free $\mathrm{Na}$ and I fragments in the electronic ground state increases in a stepwise manner, with essentially constant populations in between the outgoing passages of the curve crossing.

An interesting feature of this type of indirect reaction is that the product distributions-in the case of Eq. (1) the momentum distribution of the atomic fragments-is time dependent. This interesting phenomenon seems-with a few notable exceptions ${ }^{5-7}$ - to have attracted little attention.

The aim of the present work is to elaborate on the theoretical analysis of this phenomenon. We focus on indirect dissociation of a diatomic molecule via nonadiabatic dynam-

\footnotetext{
${ }^{a)}$ Electronic mail: neh@kemi.dtu.dk.
}

ics, as in Fig. 1. A wave packet consisting of a superposition of the resonance states is prepared by a femtosecond laser pulse, and the momentum distribution of the free fragments is analyzed at different times.

Measurement of the terminal velocity or kinetic energy of photofragments (via time-of-flight) is an important tool in order to elucidate the distribution of internal energy in molecular fragments, see, e.g., Refs. 8 and 9. More relevant in the present context, even a direct mapping of the kinetic energy along the reaction coordinate, is experimentally feasible on the femtosecond time scale. ${ }^{10}$

This paper is organized in the following way. In Sec. II, the relation between the directly measurable atomic momenta of the fragments and the relative momentum is discussed. In Sec. III, analytical results are derived for the momentum distribution in the force free region after the passage of a curve crossing. In Sec. IV numerical results for NaI are presented and discussed. Finally, conclusions and outlook are given in Sec. V.

\section{ATOMIC MOMENTA-RELATIVE MOMENTUM}

In the gas phase the overall center-of-mass motion is separable from the internal (relative) motion, the latter is therefore at the focus of most theoretical analysis. We consider here the relation between the directly measurable atomic momenta of the fragments and the relative momentum. Since this relation typically is discussed within the framework of classical mechanics, we provide here the relation within quantum mechanics.

Consider two atoms, i.e., nuclei moving in a potential $V(r)$ with associated momentum operators $\hat{\mathbf{p}}_{1}$ and $\hat{\mathbf{p}}_{2}$. After the introduction of the relative coordinates $\mathbf{r}$ and the centerof-mass coordinates $\mathbf{R}$, the total Hamiltonian can be written in the form

$$
\mathcal{H}=\frac{\hat{\mathbf{p}}^{2}}{2 \mu}+V(r)+\frac{\hat{\mathbf{P}}^{2}}{2 M},
$$

where $\mu$ is the reduced mass, $M$ is the total mass, and the relative and total momenta 


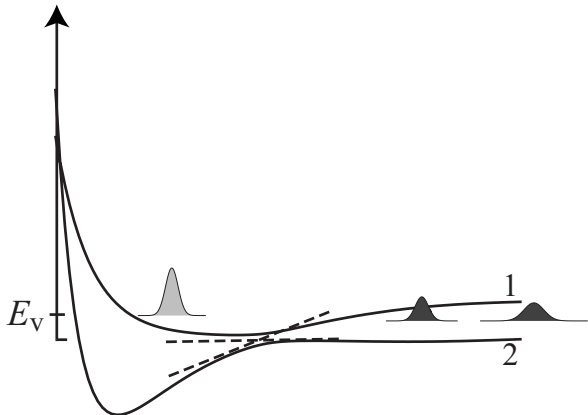

FIG. 1. Dissociation of a diatomic molecule via nonadiabatic dynamics, where free atomic fragments show up in electronic state " 2. " The laserinduced nuclear wave packet is sketched in the quasibound part of the potential and in the asymptotic force-free region, at two different times, illustrating the stepwise formation of fragments. $E_{v}$ is the vertical excitation energy. The dashed lines indicate the linearized diabatic states in the crossing region.

$$
\begin{aligned}
& \hat{\mathbf{p}}=\left(m_{2} / M\right) \hat{\mathbf{p}}_{1}-\left(m_{1} / M\right) \hat{\mathbf{p}}_{2}, \\
& \hat{\mathbf{P}}=\hat{\mathbf{p}}_{1}+\hat{\mathbf{p}}_{2}
\end{aligned}
$$

are associated with the relative coordinates and the centerof-mass coordinates, respectively. That is,

$$
\begin{aligned}
& \hat{\mathbf{p}}_{1}=\hat{\mathbf{p}}+\left(m_{1} / M\right) \hat{\mathbf{P}}, \\
& \hat{\mathbf{p}}_{2}=-\hat{\mathbf{p}}+\left(m_{2} / M\right) \hat{\mathbf{P}} .
\end{aligned}
$$

The probability of observing the momentum $\mathbf{p}_{1}$ of atom 1 at time $t$ is $P\left(\mathbf{p}_{1}, t\right) d \mathbf{p}_{1}$, where

$$
P\left(\mathbf{p}_{1}, t\right)=\left|\left\langle\mathbf{p}_{1} \mid \Psi(t)\right\rangle\right|^{2}
$$

the total wave function can be written in the form $\Psi(t)$ $=\psi_{\text {rel }}(\mathbf{r}, t) \psi_{\mathrm{CM}}(\mathbf{R}, t)$, that is, a wave function which describes the relative motion times a wave function associated with the center of mass, and the momentum eigenstates are given by $\hat{\mathbf{p}}_{1}\left|\mathbf{p}_{1}\right\rangle=\mathbf{p}_{1}\left|\mathbf{p}_{1}\right\rangle$. Then using Eq. (4), the eigenvalues can be written as

$$
\mathbf{p}_{1}=\mathbf{p}+\left(m_{1} / M\right) \mathbf{P}
$$

with eigenfunctions $\left\langle\mathbf{r}, \mathbf{R} \mid \mathbf{p}_{1}\right\rangle=\langle\mathbf{r} \mid \mathbf{p}\rangle\langle\mathbf{R} \mid \mathbf{P}\rangle$, i.e., a product of plane waves associated with the relative motion and the center-of-mass coordinates. Then, Eq. (5) takes the form

$$
P\left(\mathbf{p}_{1}, t\right)=P_{\text {rel }}(\mathbf{p}, t) P_{\mathrm{CM}}(\mathbf{P}),
$$

where the first factor is a Fourier transform (FT) of $\psi_{\text {rel }}(\mathbf{r}, t)$ and the second factor is a FT of $\psi_{\mathrm{CM}}(\mathbf{R}, t)$.

As an example, assume that the center-of-mass motion is described by a (separable) Gaussian wave packet, then

$$
P_{\mathrm{CM}}(\mathbf{P})=\prod_{i=X, Y, Z}\left(2 \pi\left(\Delta P_{i}\right)^{2}\right)^{-1 / 2} \exp \left[-\frac{\left(P_{i}-P_{i}^{\mathrm{CM}}\right)^{2}}{2\left(\Delta P_{i}\right)^{2}}\right],
$$

where $P_{i}^{\mathrm{CM}}$ and $\left(\Delta P_{i}\right)$ are, respectively, the expectation value and the standard deviation of the momentum associated with the $i$ th coordinate. Contributions to the momentum $\mathbf{p}_{1}$ [see Eq. (6)] from the center-of-mass motion can be eliminated in a molecular beam setup. Assume that detection is perpendicular to $\mathbf{P}^{\mathrm{CM}}=\left(P_{X}^{\mathrm{CM}}, 0,0\right)$, i.e, detection in the $Y Z$ plane.
Then $P_{Y} \sim 0$ and $P_{Z} \sim 0$ within the uncertainty in momentum, and $P\left(\mathbf{p}_{1}, t\right) \sim P_{\text {rel }}(\mathbf{p}, t)$.

Within first-order perturbation theory for laser-excited photofragmentation, the dynamics is governed by the molecular Hamiltonian $\hat{\mathbf{p}}^{2} / 2 \mu+V(r)$ of Eq. (2), and angular momentum is conserved due to rotational invariance. With the rotational ground state as initial state, the momentum distribution becomes a product of an angular part $\left(\propto \cos ^{2} \theta\right.$, where $\theta$ is the angle between polarization direction of the electric field of the laser and the transition dipole vector) and a radial part. ${ }^{11}$ A description and model of the latter is given in Sec. III. For initial states originating from higher rotational states, two angular momentum eigenstates contribute to the momentum distribution. Thus, in this case there is a time-dependent coupling between the magnitude of the momentum and the direction of observation. The time dependence is expected to be of the order of the magnitude of the rotational constant, with faster rotational dynamics as higher rotational states come into play.

\section{ANALYTICAL RESULTS}

In this section, we derive some analytical expressions for the probability of observing fragments at a certain relative momentum or the equivalent kinetic energy.

The electric field of the laser pulse can be represented by

$$
\mathcal{E}(t)=\mathcal{E}_{0} \int_{-\infty}^{\infty} A(\omega) e^{i \phi(\omega)} e^{-i \omega t} d \omega,
$$

where $A(\omega)$ is the real-valued distribution of frequencies and $\phi(\omega)$ is the real-valued frequency-dependent phase. We consider in the following the situation where the phases are constant, e.g., $\phi(\omega)=0$ and a transform-limited (Gaussian) pulse.

An electronic transition in a molecule, from electronic state " 0 " to " 1 " is considered. Within the electric-dipole approximation and first-order perturbation theory for the interaction with the electromagnetic field, the state vector associated with the nuclear motion in electronic state 1 is, after the pulse has decayed, given by (see, e.g., Ref. 12)

$$
\left|\psi_{1}(t)\right\rangle=\frac{i \pi}{\hbar} \sum_{n} c_{n}\left|E_{n}\right\rangle e^{-i E_{n} t / \hbar}
$$

where excitation into nuclear eigenstates $\left|E_{n}\right\rangle$ associated with a bound electronic state has been assumed. The expansion coefficients are given by

$$
c_{n}=\mathcal{E}_{0} A\left(\omega_{E_{n}}\right)\left\langle E_{n}\left|\mu_{01}\right| \psi_{0}\right\rangle
$$

where $\omega_{E_{n}}=\left(E_{n}-\epsilon_{0}\right) / \hbar, \mu_{01}$ is the transition-dipole moment, and $\left|\psi_{0}\right\rangle$ is the initial vibrational eigenstate. Thus, the expansion coefficients of the wave packet are a product of the frequency distribution of the laser pulse and Franck-Condon factors.

The laser-excited state is now subject to nonadiabatic dynamics described by 


$$
i \hbar \frac{\partial}{\partial t}\left[\begin{array}{l}
\psi_{1}(r, t) \\
\psi_{2}(r, t)
\end{array}\right]=\left[\begin{array}{ll}
\hat{H}_{1} & \hat{C}_{12} \\
\hat{C}_{21} & \hat{H}_{2}
\end{array}\right]\left[\begin{array}{l}
\psi_{1}(r, t) \\
\psi_{2}(r, t)
\end{array}\right],
$$

where $\hat{H}_{i}$ is the nuclear Hamiltonian associated with the $i$ th adiabatic electronic state, $\hat{C}_{i j}$ are nonadiabatic coupling operators, and $r$ denotes nuclear coordinates.

When the wave packet approaches the coupling region, see Fig. 1, it is convenient to switch to the diabatic representation. Assuming weak coupling, first-order perturbation theory gives

$$
\left|\psi_{2}(t)\right\rangle=-\frac{i}{\hbar} \int_{0}^{t} d t^{\prime} \hat{U}_{2}\left(t, t^{\prime}\right) V_{21}\left|\psi_{1}\left(t^{\prime}\right)\right\rangle,
$$

where $\left|\psi_{1}(t)\right\rangle$ is the laser excited state and $V_{21}$ is the scalar coupling element in the diabatic representation. The situation corresponds now to the decay of a coherent superposition of resonance states. The decay is due to coupling to the continuum.

Furthermore, from the example in Fig. 1, $\hat{U}_{2}\left(t, t^{\prime}\right)$ is to a good approximation the propagator for a free particle

$$
\begin{aligned}
\hat{U}_{2}\left(t, t^{\prime}\right) & =e^{-i \hat{H}_{2}\left(t-t^{\prime}\right) / \hbar} \int_{0}^{\infty} d E|E, p\rangle\langle E, p| \\
& =\int_{0}^{\infty} d E|E, p\rangle e^{-i E\left(t-t^{\prime}\right) / \hbar}\langle E, p|,
\end{aligned}
$$

where $|E, p\rangle$ is an eigenstate corresponding to free relative motion at energy $E \equiv E_{p}=p^{2} /(2 \mu)$, with a definite relative momentum $p$ (in the following, $p \geq 0$, corresponding to recoiling fragments).

The probability of observing fragments at the relative kinetic energy $E_{0} \equiv E_{p_{0}}=p_{0}^{2} /(2 \mu)$, is

$$
P\left(E_{0}, t\right) d E_{0}=\left|\left\langle E_{0}, p_{0} \mid \psi_{2}(t)\right\rangle\right|^{2} d E_{0},
$$

where, assuming for simplicity that $V_{21}$ is constant,

$$
\begin{aligned}
\left|\left\langle E_{0}, p_{0} \mid \psi_{2}(t)\right\rangle\right|^{2} /\left(\pi^{2}\left|V_{21}\right|^{2}\right) & \left|\sum_{n} c_{n}\left\langle E_{0}, p_{0} \mid E_{n}\right\rangle \frac{e^{-i\left(E_{n}-E_{p_{0}}\right) t / \hbar}-1}{\left(E_{n}-E_{p_{0}}\right) / \hbar}\right|^{2} \\
= & c_{1}^{2}\left|\left\langle E_{0}, p_{0} \mid E_{1}\right\rangle\right|^{2}\left[f\left(E_{1}-E_{p_{0}}\right)\right]^{2}+c_{2}^{2}\left|\left\langle E_{0}, p_{0} \mid E_{2}\right\rangle\right|^{2} \\
& \quad \times\left[f\left(E_{2}-E_{p_{0}}\right)\right]^{2}+2 \operatorname{Re}\left\{c_{1} c_{2}^{*}\left\langle E_{0}, p_{0} \mid E_{1}\right\rangle\left\langle E_{0}, p_{0} \mid E_{2}\right\rangle^{*}\right. \\
& \left.\quad \times f\left(E_{1}-E_{p_{0}}\right) f\left(E_{2}-E_{p_{0}}\right) e^{-i\left(E_{1}-E_{2}\right) t / 2 \hbar}\right\}
\end{aligned}
$$

using $\left\langle E_{0}, p_{0} \mid E, p\right\rangle=\delta\left(E_{p_{0}}-E_{p}\right)$, i.e., energy normalization and the sum was specialized to excitation of two states $\left|E_{1}\right\rangle$ and $\left|E_{2}\right\rangle$, and

$$
f\left(E_{n}-E_{p_{0}}\right)=\frac{\sin \left[\left(E_{n}-E_{p_{0}}\right) t / 2 \hbar\right]}{\left[\left(E_{n}-E_{p_{0}}\right) / 2 \hbar\right]}
$$

is peaked around $E_{n}=E_{p_{0}}$. Thus, $\left[f\left(E_{n}-E_{p_{0}}\right)\right]^{2} \rightarrow 2 \pi \hbar \delta\left(E_{n}\right.$ $\left.-E_{p_{0}}\right) t$ for $t \rightarrow \infty$, i.e., for sufficiently long times, but not too long to invalidate the perturbation treatment, that is, the energy conservation expressed by the delta function is not fully realistic. A more accurate description beyond first-order per-

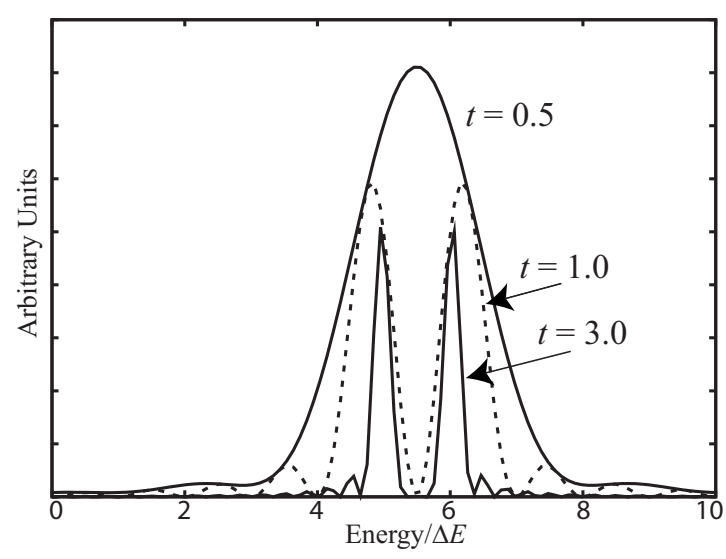

FIG. 2. Probability distribution for the relative energy of fragments according to Eq. (16) (see text for details). The energy is in units of the energy spacing $\Delta E=E_{1}-E_{2}$ of the two states and time $t$ is measured in units of the associated vibrational period. The values of the maxima increase as a function of time but are here, for graphical purpose, rescaled.

turbation theory, gives a Lorentzian energy dependence for the decay of a single isolated resonance state $\left|E_{i}\right\rangle .{ }^{13}$ However, it should be noted that the decay of a set of coherently excited resonances is coupled via the continuum, ${ }^{4,14}$ i.e., strictly speaking the states do not decay independently.

Equation (16) suggests that the momentum distribution will consist of peaks centered at $E_{1}, E_{2}, \cdots$ weighted by the expansion coefficients $c_{1}, c_{2}, \cdots$ and the FTs of $\left|E_{1}\right\rangle,\left|E_{2}\right\rangle, \cdots$ i.e., the momentum representation of the states. The last term is an interference term. This term is clearly in play for overlapping resonances, i.e., when the resonances are wider than the adjacent level spacing $\left|E_{1}-E_{2}\right|$, that is, $f\left(E_{1}-E_{p_{0}}\right) f\left(E_{2}\right.$ $\left.-E_{p_{0}}\right) \neq 0$. The coupling to the dissociative electronic state acts as a "time-energy" filter. Thus, at short times after the first passage of the crossing, the uncertainty in the relative energy of the emerging products is large. At longer times, after additional passages of the coupling region, signatures of well-defined energy levels show up. Figure 2 illustrates Eq. (16), where for simplicity it is assumed that only two states are excited and that the expansion coefficients $c_{i}$ as well as $\left\langle E_{0}, p_{0} \mid E_{i}\right\rangle$ are identical for the two states.

In the following, we derive a more explicit analytical expression. In the first, outgoing, passage of the crossing the relevant dynamics of $\left|\psi_{1}(t)\right\rangle$ takes roughly place in a purely repulsive potential. We replace Eq. (10) by

$$
\left|\psi_{1}(t)\right\rangle=\frac{i \pi}{\hbar} \int d E c_{E}|E\rangle e^{-i E t / \hbar},
$$

where excitation into continuum states $|E\rangle$ associated with a repulsive electronic state is assumed. The expansion coefficients are given by $c_{E}=\mathcal{E}_{0} A\left(\omega_{E}\right)\left\langle E\left|\mu_{01}\right| \psi_{0}\right\rangle$ where $\omega_{E}$ $=\left(E-\epsilon_{0}\right) / \hbar$ and $\left|\psi_{0}\right\rangle$ is the initial vibrational eigenstate. Equation (16) takes then the form

$$
\begin{aligned}
& \left|\left\langle E_{0}, p_{0} \mid \psi_{2}(t)\right\rangle\right|^{2} \\
& \quad=\frac{\pi^{2}}{\hbar^{2}}\left|V_{21}\right|^{2}\left|\int_{0}^{\infty} d E c_{E}\left\langle E_{0}, p_{0} \mid E\right\rangle \frac{e^{-i\left(E-E_{0}\right) t / \hbar}-1}{E-E_{0}}\right|^{2} .
\end{aligned}
$$

It should be recalled that the states $|E\rangle$ and $\left|E_{0}, p_{0}\right\rangle$ are asso- 
ciated with different Hamiltonians, thus $\left\langle E_{0}, p_{0} \mid E\right\rangle$ is not subject to an orthonormality condition.

In order to proceed, we invoke the Landau-Zener model. ${ }^{15,16} V_{21}$ is the constant scalar coupling element between linearized diabatic potentials. Representing the repulsive potential by a linear potential $V_{1}=-f x$, the energy eigenstates $|E\rangle$ take in the (one-dimensional) momentum representation the form

$$
\langle p \mid E\rangle=(2 \pi \hbar f)^{-1 / 2} \exp \left[-\frac{i}{\hbar f}\left(\frac{p^{3}}{6 \mu}-E p\right)\right],
$$

where the normalization constant is chosen such that $\left\langle E \mid E^{\prime}\right\rangle=\delta\left(E-E^{\prime}\right)$. We note that $\langle E, p \mid E\rangle=\langle p \mid E\rangle \sqrt{\mu / p}$ (energy to momentum normalization). It is convenient to use the identity

$$
\frac{e^{-i\left(E-E_{0}\right) t / \hbar}-1}{E-E_{0}}=-i e^{-i E^{\prime} t / 2 \hbar} \frac{\sin \left[E^{\prime} t / 2 \hbar\right]}{E^{\prime} / 2},
$$

where $E^{\prime}=E-E_{0}$ and we note that the last factor is peaked around $E=E_{0}$ (its absolute square approach a delta function for $t \rightarrow \infty)$. Then,

$$
\begin{aligned}
\left|\left\langle E_{0}, p_{0} \mid \psi_{2}(t)\right\rangle\right|^{2}= & \frac{2 \pi\left|V_{21}\right|^{2}}{\hbar|f|\left(p_{0} / \mu\right)} \frac{1}{4 \hbar^{2}} \mid \int_{-\infty}^{\infty} d E^{\prime} e^{i\left(p_{0} / f\right) E^{\prime} / \hbar} c_{E^{\prime}} \\
& \times\left. e^{-i E^{\prime} t / 2 \hbar} \frac{\sin \left[E^{\prime} t / 2 \hbar\right]}{E^{\prime} / 2}\right|^{2}
\end{aligned}
$$

where the lower limit has been extended to $-\infty$, since $c_{E^{\prime}}$ is nonzero only for positive values of the energy.

The first factor is the "adiabaticity parameter" of the Landau-Zener theory, corresponding to a small transition probability between the diabatic states and, consequently, a large transition probability between the adiabatic states.

The integral in Eq. (22) can be viewed as a FT of a product of two functions, and evaluated as the FT of $c_{E^{\prime}}$ convoluted with the FT of the last two factors. In the limit $t \rightarrow \infty$, we get $^{17}$

$$
\int_{-\infty}^{\infty} d E^{\prime} e^{i\left(p_{0} / f\right) E^{\prime} / \hbar} e^{-i E^{\prime} t / 2 \hbar} \frac{\sin \left[E^{\prime} t / 2 \hbar\right]}{E^{\prime} / 2}=(1 / 2) \theta(y),
$$

where $\theta(y)$ is the Heaviside unit step function and $y$ $=p_{0} /(\hbar f)$. Thus, the FT in Eq. (22) becomes $(1 / 4 \pi) \int_{0}^{\infty} \mathrm{FT}\left\{c_{E^{\prime}}\right\}\left(y-y_{1}\right) d y_{1}$.

A fair approximation to the expansion coefficients in Eq. (18) is

$$
c_{E} \sim A_{0} \exp \left[-\left(E-E_{v}\right)^{2} / \sigma^{2}\right]=A_{0} \exp \left[-\left(E^{\prime}-E_{d}\right)^{2} / \sigma^{2}\right],
$$

where $E_{d}=E_{v}-E_{0}$ and $E_{v}$ is an energy determined by the mean photon energy of the laser and the maximum in the Franck-Condon factors, i.e., $E_{v}$ is in the neighborhood of the potential energy corresponding to a vertical excitation (note that the unit of $A_{0}$ is $\mathrm{J}^{1 / 2} \mathrm{~s}$ ). The width $\sigma$ reflects the energy dependence of the Franck-Condon factors as well as the frequency distribution of the laser pulse.

The probability distribution becomes then, according to Eqs. (15) and (22),
$P\left(E_{0}\right) d E_{0}=\frac{2 \pi\left|V_{21}\right|^{2}}{\hbar|f|\left(p_{0} / \mu\right)} \frac{A_{0}^{2}}{16 \hbar^{2}} \exp \left\{-2\left(E_{0}-E_{v}\right)^{2} / \sigma^{2}\right\} d E_{0}$.

The last factor is a simple Gaussian distribution in $E_{0} \equiv E_{p_{0}}$ $=p_{0}^{2} / 2 \mu$, corresponding to the very reasonable result that the most probable relative kinetic energy equals the potential energy $E_{v}$ (see Fig. 1).

Finally, we can easily convert Eq. (25) into a probability distribution in the relative momentum, $P\left(p_{0}\right) d p_{0}$, by making the substitutions $E_{0}=p_{0}^{2} / 2 \mu$ and $d E_{0}=\left(p_{0} / \mu\right) d p_{0}$ on the right-hand side of Eq. (25). Thus, $P\left(p_{0}\right) d p_{0}$ becomes a Gaussian distribution in $p_{0}^{2}$. Note that the velocity dependence $\left(p_{0} / \mu\right)$ in the pre-exponential factor of Eq. (25) is absent in the momentum distribution. The connection to the distribution of the momenta of the atomic fragments was discussed in relation to Eq. (7).

\section{NUMERICAL RESULTS FOR NaI}

In this section we present numerical results for the fragmentation of NaI, as described in Eq. (1), and we compare to the analytical predictions of Sec. III. The numerical results are similar to previous work. ${ }^{6,7}$

All calculations are performed using the wave-packet program in Ref. 18 and the potential energy curves are from Ref. 3. The calculation describes the one-dimensional relative motion, e.g., corresponding to observation along the polarization direction of the electric field of the laser. For excitation out of rotationally excited states there is, as pointed out in Sec. II, a coupling between the radial and the angular dynamics. For short times of the order of a few picoseconds (the basic rotational period- $2 B$-of $\mathrm{NaI}$ is $138 \mathrm{ps}$ ), little dependence on angular dynamics is expected.

We consider the momentum distribution of the fragments, at different times, using a Gaussian (transformlimited) pump pulse of temporal duration [full width at half maximum (FWHM)] of $30 \mathrm{fs}$. The results are shown at 0.5 and 3 ps in Fig. 3. The envelope of the momentum distribution is Gaussian-like at all times which reflects the frequency distribution of the laser pulse and the Franck-Condon factors. There is, in addition, a characteristic time-energy "filtering"-more and more features show up as time increases, exactly as in Fig. 2. However, in the present case many resonance states are excited.

The momentum distribution after $0.5 \mathrm{ps}$ - corresponding to one outgoing passage of the curve crossing-resembles the result in Fig. 2 after half a vibrational period and, in particular, the result in Eq. (25). Thus, the overall shape of the distribution and the position of the center at $p_{0}=\sqrt{2 \mu E_{v}}$ is as predicted by Eq. (25). To be more specific, the relative momentum corresponding to the mean photon energy $(\lambda$ $=328 \mathrm{~nm}$ ) of the excitation is $p \sim 41$ a.u. This mean photon energy is, however, slightly below the vertical excitation energy where the Franck-Condon factors are expected to take their maximum value. This explains/justifies that $p_{0}$ $\sim 42$ a.u. in the numerical result.

After about 1-2 ps clear signatures of the quasibound vibrational resonance states are found in agreement with the diagonal terms in Eq. (16). The spacing between the lines 


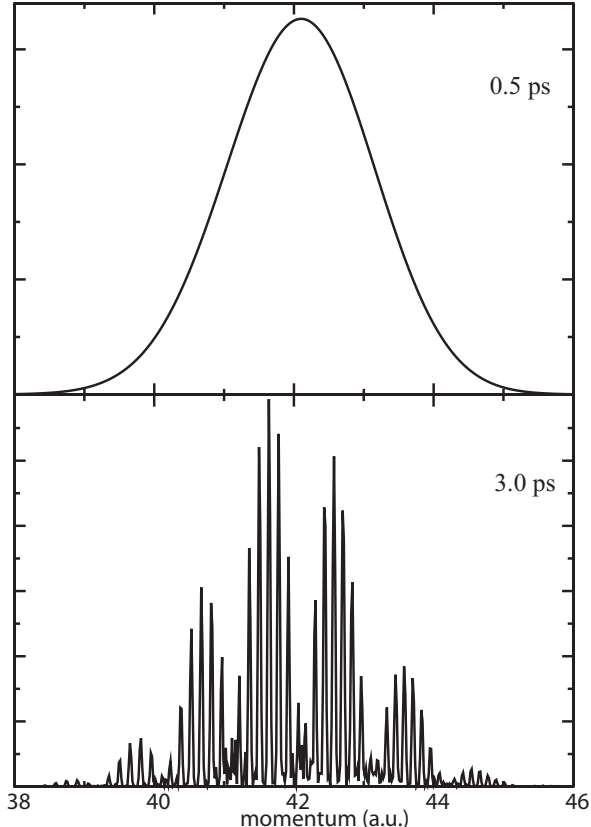

FIG. 3. Probability distribution for relative momentum (in atomic units) of $\mathrm{Na}+\mathrm{I}$, after 0.5 and $3.0 \mathrm{ps}$, respectively. A Gaussian pump pulse of temporal duration (FWHM) $30 \mathrm{fs}$ and center wavelength $328 \mathrm{~nm}$ is employed.

around the center corresponds to $\sim 35 \mathrm{~cm}^{-1}$, and this is exactly the known spacing between the quasibound energy levels ${ }^{19}$ of $\mathrm{NaI}$.

The peak heights show a regular alternation between high and low, similar to the absorption spectrum of $\mathrm{NaI}$ where the dependence on the lifetimes of the quasibound resonance states leads to a regular alternation between broad and narrow lines (as in Fig. 4 of Ref. 19).

Momentum distributions can be observed experimentally at various delay times on the femtosecond time scale, see, e.g., Ref. 10 where a probe pulse freezes the distribution via excitation to a state where there is no forces between the recoiling atoms. A theoretical analysis of the detection via pump-probe ionization has also been worked out. ${ }^{7}$

\section{CONCLUSIONS}

The aim of this work was to highlight and supplement the previous numerical observations ${ }^{6,7}$ of time-dependent fragment distributions in indirect photofragmentation with a theoretical analysis of this phenomenon. For completeness, we considered first the relation between the measurable atomic momenta and the relative momentum of a diatomic molecule, within a quantum mechanical framework. We presented a theoretical analysis of the distribution of relative momentum after the dissociation via a curve crossing. At short times, after a single passage of the crossing, we obtained an expression for the associated distribution of rela- tive kinetic energies Eq. (25) which, e.g., contains a factor identical to the "adiabaticity parameter" in the associated Landau-Zener transition probability. The general predictions of the analytical expressions are in agreement with the numerical results for the time-dependent (transient) momentum distribution in the fragmentation of NaI.

An interesting question is whether-in the weak-field limit-the final product distribution might be changed by employing a phase modulated excitation pulse [see Eq. (9)]. That is, if pulse shaping (disregarding trivial affects of varying the frequency distribution of the pulse) can change the momentum distribution of the fragments. For direct fragmentation, in the long-time limit, it has been shown that such control is not possible. ${ }^{20}$ In the context of indirect fragmentation via a coherent superposition of resonance states, as studied in this paper, one might anticipate that it is possible to modify the (transient) distributions at finite times. Thus, it is, e.g., well known that linearly chirped pulses can delay or speed up the arrival of the various momentum components.

\section{ACKNOWLEDGMENTS}

Ulf Lorenz is thanked for performing the numerical calculations in Fig. 3. The Danish National Research Foundation's Center for Molecular Movies (CMM) is acknowledged for financial support.

${ }^{1}$ T. S. Rose, M. J. Rosker, and A. H. Zewail, J. Chem. Phys. 88, 6672 (1988).

${ }^{2}$ V. Engel, H. Metiu, R. Almeida, R. A. Marcus, and A. H. Zewail, Chem. Phys. Lett. 152, 1 (1988).

${ }^{3}$ T. S. Rose, M. J. Rosker, and A. H. Zewail, J. Chem. Phys. 91, 7415 (1989).

${ }^{4}$ K. B. Møller, N. E. Henriksen, and A. H. Zewail, J. Chem. Phys. 113, 10477 (2000)

${ }^{5}$ V. Engel and H. Metiu, J. Chem. Phys. 92, 2317 (1990).

${ }^{6}$ C. Meier, V. Engel, and J. S. Briggs, J. Chem. Phys. 95, 7337 (1991).

${ }^{7}$ J. Degert, C. Meier, B. Girard, and M. J. J. Vrakking, Eur. Phys. J. D 14, 257 (2001).

${ }^{8}$ G. E. Busch and K. R. Wilson, J. Chem. Phys. 56, 3626 (1972).

${ }^{9}$ D. W. Hwang, X. Yang, and X. Yang, J. Chem. Phys. 110, 4119 (1999).

${ }^{10}$ A. V. Baklanov, L. M. C. Janssen, D. H. Parker, L. Poisson, B. Soep, J.-M. Mestdagh, and O. Gobert, J. Chem. Phys. 129, 214306 (2008).

${ }^{11}$ N. E. Henriksen, Adv. Chem. Phys. 91, 433 (1995).

${ }^{12}$ N. E. Henriksen and K. B. Møller, J. Chem. Phys. 119, 2569 (2003).

${ }^{13}$ R. Schinke, Photodissociation Dynamics (Cambridge University Press, London, 1993).

${ }^{14}$ M. Bixon and J. Jortner, J. Chem. Phys. 107, 1470 (1997).

${ }^{15}$ R. D. Levine, Molecular Reaction Dynamics (Cambridge University Press, Cambridge, 2005).

${ }^{16}$ N. E. Henriksen, Chem. Phys. Lett. 197, 620 (1992).

${ }^{17}$ D. C. Champeney, Fourier Transforms and Their Physical Applications (Academic, New York, 1973).

${ }^{18}$ U. Lorenz and B. Schmidt, Wave packet 4.7.2: a program package for quantum-mechanical wave packet propagation and time-dependent spectroscopy http://wavepacket.sourceforge.net (2010).

${ }^{19}$ S. Chapman and M. S. Child, J. Phys. Chem. 95, 578 (1991).

${ }^{20}$ P. Brumer and M. Shapiro, Chem. Phys. 139, 221 (1989). 